\title{
Positive Solutions of the Semipositone Neumann Boundary Value Problem
}

\section{Johnny Henderson ${ }^{a}$ and Nickolai Kosmatov ${ }^{b}$}

${ }^{a}$ Baylor University, Department of Mathematics

Waco, 76798-7328 TX, USA

${ }^{b}$ University of Arkansas at Little Rock, Department of Mathematics and Statistics

Little Rock, 72204-1099 AR, USA

E-mail: Johnny_Henderson@baylor.edu

E-mail(corresp.): nxkosmatov@ualr.edu

Received September 1, 2014; revised August 3, 2015; published online September 15, 2015

Abstract. In this paper we consider the Neumann boundary value problem at resonance

$$
-u^{\prime \prime}(t)=f(t, u(t)), \quad 0<t<1, \quad u^{\prime}(0)=u^{\prime}(1)=0 .
$$

We assume that the nonlinear term satisfies the inequality $f(t, z)+\alpha^{2} z+\beta(t) \geq 0$, $t \in[0,1], z \geq 0$, where $\beta:[0,1] \rightarrow \mathbf{R}_{+}$, and $\alpha \neq 0$. The problem is transformed into a non-resonant positone problem and positive solutions are obtained by means of a Guo-Krasnosel'skiĭ fixed point theorem.

Keywords: Neumann boundary conditions, resonance, semipositone.

AMS Subject Classification: 34B15; 34B18.

\section{Introduction}

We study the Neumann boundary value problem

$$
\begin{gathered}
-u^{\prime \prime}(t)=f(t, u(t)), \quad 0<t<1, \\
u^{\prime}(0)=u^{\prime}(1)=0
\end{gathered}
$$

with a sign-changing nonlinearity.

We will make the assumptions precise in the next section, we only mention now that the continuous function $f:[0,1] \times \mathbf{R}_{+} \rightarrow \mathbf{R}$ satisfies the inequality $f(t, z) \geq-\alpha^{2} z-\beta(t)$ in $[0,1] \times \mathbf{R}_{+}$, for some constant $\alpha \neq 0$ and a non-negative valued function $\beta(t)$.

One of the most frequently mentioned papers that stimulated the discussion of semipositone problems is the paper [7] by Miciano and Shivaji. The authors 
of [7] used the bifurcation techniques to obtain multiple positive solutions for the Neumann problem. We only mention several among many results based on applications of a Guo-Krasnosel'skiı fixed point theorem and fixed point index computations. In [10], Sun and Wei obtained positive solutions of the non-local boundary value problem

$$
\begin{gathered}
-u^{\prime \prime}(t)=f(t, u(t)), \quad 0<t<1, \\
u(0)=\alpha u(\eta), \quad u(1)=\beta u(\eta),
\end{gathered}
$$

where the right side is a continuous function with $f(t, u)+M \geq 0$ for some $M>0$. Lu [5] obtained multiple positive solutions for singular semipositone periodic boundary value problems. It should be mentioned that, in [5], the nonhomogeneous term depends on the first order derivative. In this regard, the results of [5] are similar to those obtained by Ma [6] who studied a fourth order semipositone boundary value problem

$$
\begin{aligned}
u^{(4)}(t) & =\lambda f\left(t, u(t), u^{\prime}(t)\right), \quad 0<t<1, \\
u(0) & =u^{\prime}(0)=u^{\prime \prime}(1)=u^{\prime \prime \prime}(1)=0 .
\end{aligned}
$$

Other interesting results for second order boundary value problems can be found in $[1,4,9,13]$. Semipositone boundary value problems of higher order have been studied in $[2,6,11,12]$ just to name a few. It seems, however, that resonant semipositone problems for ordinary differential equations have not been studied as extensively as their "invertible" counterparts. Nkashama and Santanilla [8] obtained nonpositive and nonnegative solutions of the Neumann problem using generalized Ambrosetti-Prodi conditions. Since we are unaware of results based on cone-theoretic methods, we believe that our study of the Neumann problem provides new results. We only treat the most basic case of (1.1) with a continuous right side.

\section{Properties of Green's Function}

As a first step, we introduce $g(t, z)=f(t, z)+\alpha^{2} z$ to transform (1.1) into

$$
-u^{\prime \prime}(t)+\alpha^{2} u(t)=g(t, u(t)), \quad t \in(0,1),
$$

which we consider together with the boundary condition (1.2).

For $\beta \in C[0,1]$, the differential equation

$$
-u^{\prime \prime}(t)+\alpha^{2} u(t)=\beta(t), \quad 0<t<1,
$$

satisfying the boundary condition (1.2) has a unique solution

$$
u_{0}(t)=\int_{0}^{1} G(t, s) \beta(s) d s
$$

with the Green function

$$
G(t, s)=\frac{1}{\alpha \sinh \alpha} \begin{cases}\cosh \alpha(1-t) \cosh \alpha s, & 0 \leq s \leq t \leq 1 \\ \cosh \alpha t \cosh \alpha(1-s), & 0 \leq t \leq s \leq 1\end{cases}
$$


It is obvious that

$$
G(t, s) \leq G(s, s), \quad(t, s) \in[0,1] \times[0,1] .
$$

If $s \leq t$, then

$$
\begin{aligned}
G(t, s) & =\frac{1}{\alpha \sinh \alpha} \cosh \alpha(1-t) \cosh \alpha s \\
& \geq \frac{1}{\alpha \sinh \alpha} \cosh \alpha(1-t) \cosh \alpha s \frac{\cosh \alpha(1-s)}{\cosh \alpha} \\
& \geq \frac{\cosh \alpha(1-t)}{\cosh \alpha} G(s, s) .
\end{aligned}
$$

Similarly, for $t \leq s$,

$$
G(t, s) \geq \frac{\cosh \alpha t}{\cosh \alpha} G(s, s) .
$$

Combining the inequalities above, we obtain

$$
q(t) G(s, s) \leq G(t, s) \leq G(s, s), \quad(t, s) \in[0,1] \times[0,1],
$$

where

$$
q(t)=\frac{1}{\cosh \alpha} \min \{\cosh \alpha t, \cosh \alpha(1-t)\}
$$

Also,

$$
L=\max _{t \in[0,1]} \int_{0}^{1} G(t, s) d s=\frac{1}{\alpha^{2}}
$$

For $0<\gamma<1 / 2$,

$$
\int_{\gamma}^{1-\gamma} G(1-t, s) d s=\int_{\gamma}^{1-\gamma} G(1-t, 1-s) d s=\int_{\gamma}^{1-\gamma} G(t, s) d s .
$$

It suffices to consider

$$
\begin{aligned}
& \int_{\gamma}^{1-\gamma} G(t, s) d s \\
& \quad=\frac{1}{\alpha^{2} \sinh \alpha} \begin{cases}(\sinh \alpha(1-\gamma)-\sinh \alpha \gamma) \cosh \alpha t, & 0 \leq t \leq \gamma, \\
\sinh \alpha-\sinh \alpha \gamma(\cosh \alpha t+\cosh \alpha(1-t)), & \gamma \leq t \leq 1 / 2,\end{cases}
\end{aligned}
$$

for $t \in[0,1 / 2]$, since the above function is symmetric about $t=1 / 2$. Since it is increasing in $[0,1 / 2]$,

$$
C=\max _{t \in[0,1]} \int_{\gamma}^{1-\gamma} G(t, s) d s=\frac{1}{\alpha^{2} \sinh \alpha}(\sinh \alpha-2 \sinh \alpha \gamma \cosh \alpha / 2) .
$$

Lemma 1. Let $\beta \in C[0,1]$ and $\beta(t) \geq 0$ in $[0,1], \beta(\tau)>0$ for some $\tau \in[0,1]$. Then the inequality

$$
q(t) \geq \mu u_{0}(t), \quad t \in[0,1]
$$

holds for

$$
\mu=\frac{\alpha \sinh \alpha}{\cosh ^{2} \alpha \int_{0}^{1} \beta(s) d s}
$$


Proof. Note that

$$
u_{0}(t)=\int_{0}^{1} G(t, s) \beta(s) d s \leq G(t, t) \int_{0}^{1} \beta(s) d s .
$$

Hence

$$
\begin{aligned}
q(t) & =\frac{1}{\cosh \alpha} \min \{\cosh \alpha t, \cosh \alpha(1-t)\} \\
& \geq \min \left\{\frac{\cosh \alpha t}{\cosh \alpha}, \frac{\cosh \alpha(1-t)}{\cosh \alpha}\right\} \frac{1}{\cosh \alpha} \max \{\cosh \alpha t, \cosh \alpha(1-t)\} \\
& =\frac{1}{\cosh ^{2} \alpha} \cosh \alpha t \cosh \alpha(1-t)=\frac{\alpha \sinh \alpha}{\cosh ^{2} \alpha} G(t, t) \\
& =\mu G(t, t) \int_{0}^{1} \beta(s) d s \geq \mu u_{0}(t)
\end{aligned}
$$

for all $t \in[0,1]$.

Suppose that the function $f$ in (1.1) satisfies

(A) $f \in C\left([0,1] \times \mathbf{R}_{+}, \mathbf{R}\right)$;

(B) there exists a function $\beta \in C[0,1], \beta(t) \geq 0$ in $[0,1], \beta(\tau)>0$ for some $\tau \in[0,1]$, and $\alpha \in \mathbf{R}, \alpha \neq 0$, such that

$$
f(t, z)+\alpha^{2} z+\beta(t) \geq 0, \quad(t, z) \in[0,1] \times \mathbf{R}_{+} .
$$

We turn our attention to the equation

$$
-v^{\prime \prime}(t)+\alpha^{2} v(t)=f_{p}\left(t, v(t)-u_{0}(t)\right), \quad t \in(0,1),
$$

where

$$
f_{p}(t, z)= \begin{cases}f(t, z)+\alpha^{2} z+\beta(t), & (t, z) \in[0,1] \times(0, \infty) \\ f(t, 0)+\beta(t), & (t, z) \in[0,1] \times(-\infty, 0]\end{cases}
$$

and impose the boundary conditions (1.2).

Definition 1. A positive solution of the boundary value problem (1.1), (1.2) is a function $u \in C^{2}[0,1]$ satisfying $(1.1),(1.2)$ and such that $u(t)>0$ in $[0,1]$.

The next lemma discusses the relationship between the problems (1.1), (1.2) and (2.10), (1.2) by means of a "shift" $u \mapsto u+u_{0}$ applied to the equation (2.1).

Lemma 2. The function $u$ is a positive solution of the boundary value problem (1.1), (1.2) if and only if the function $v=u+u_{0}$, where $u_{0}$ is given by $(2.2)$, is a solution of the boundary value problem (2.10), (1.2) satisfying $v(t)>u_{0}(t)$ in $(0,1)$. 
In the Banach space $\mathcal{B}=C[0,1]$ endowed with usual max-norm, we consider the operator

$$
T v(t)=\int_{0}^{1} G(t, s) f_{p}\left(s, v(s)-u_{0}(s)\right) d s,
$$

where $G(t, s)$ is given by $(2.3)$. By $(\mathrm{A}), T: \mathcal{B} \rightarrow \mathcal{B}$ is completely continuous.

Using the function $q$ defined by $(2.5)$, we introduce the cone

$$
\mathcal{C}=\{v \in \mathcal{B}: v(t) \geq q(t)\|v\|, t \in[0,1]\} .
$$

By (2.4), $T: \mathcal{C} \rightarrow \mathcal{C}$. One can easily confirm that a fixed point of $T$ in $\mathcal{C}$ is a solution of (2.10), (1.2), and conversely. In particular, for $0<\gamma<1 / 2$,

$$
v(t) \geq \rho\|v\|, \quad t \in[\gamma, 1-\gamma],
$$

where

$$
\rho=\min _{t \in[\gamma, 1-\gamma]} q(t)=\frac{\cosh \alpha \gamma}{\cosh \alpha}
$$

The following is a fixed point theorem due to Guo and Krasnosel'skiı.

Theorem 1. [3] Let $\mathcal{B}$ be a Banach space and let $\mathcal{C} \subset \mathcal{B}$ be a cone in $\mathcal{B}$. Assume that $\Omega_{1}, \Omega_{2}$ are open with $0 \in \Omega_{1}, \bar{\Omega}_{1} \subset \Omega_{2}$, and let

$$
T: \mathcal{C} \cap\left(\bar{\Omega}_{2} \backslash \Omega_{1}\right) \rightarrow \mathcal{C}
$$

be a completely continuous operator such that either

(i) $\|T u\| \leq\|u\|, u \in \mathcal{C} \cap \partial \Omega_{1}$, and $\|T u\| \geq\|u\|, u \in \mathcal{C} \cap \partial \Omega_{2}$, or

(ii) $\|T u\| \geq\|u\|, u \in \mathcal{C} \cap \partial \Omega_{1}$, and $\|T u\| \leq\|u\|, u \in \mathcal{C} \cap \partial \Omega_{2}$.

Then $T$ has a fixed point in $\mathcal{C} \cap\left(\bar{\Omega}_{2} \backslash \Omega_{1}\right)$.

\section{Positive Solutions}

To make use of Theorem 1, we introduce, following [11], the "height" functions $\phi, \psi: \mathbf{R}_{+} \rightarrow \mathbf{R}_{+}$defined by

$$
\begin{aligned}
& \phi(r)=\max \left\{f_{p}\left(t, z-u_{0}(t)\right): t \in[0,1], z \in[0, r]\right\} \\
& \psi(r)=\min \left\{f_{p}\left(t, z-u_{0}(t)\right): t \in[\gamma, 1-\gamma], z \in[\rho r, r]\right\}, \quad 0<\gamma<1 / 2 .
\end{aligned}
$$

We present our main results.

Theorem 2. Assume that (A) and (B) hold. Suppose that there exist $r, R>0$ such that $\frac{1}{\mu}<r<R$, where $\mu>0$ satisfies (2.8), (2.9), and

(C) $\phi(r) \leq \alpha^{2} r$ and $\psi(R) \geq \frac{\alpha^{2} \sinh \alpha}{\sinh \alpha-2 \sinh \alpha \gamma \cosh \alpha / 2} R$.

Then the boundary value problem (1.1), (1.2) has at least one positive solution. 
Proof. Let

$$
\Omega_{1}=\{v \in \mathcal{B}:\|v\|<r\} \quad \text { and } \quad \Omega_{2}=\{v \in \mathcal{B}:\|v\|<R\} .
$$

For $v \in \mathcal{C} \cap \partial \Omega_{1}$, by Lemma 1 , we have

$$
v(s)-u_{0}(s) \geq q(s)\|v\|-u_{0}(s) \geq(\mu r-1) u_{0}(s)>0, s \in[0,1] .
$$

This implies that $f_{p}\left(s, v(s)-u_{0}(s)\right) \leq \phi(r)$, for $s \in[0,1], 0 \leq v(s) \leq r$. Thus, by $(2.6)$ and $(\mathrm{C})$,

$$
\begin{aligned}
\|T v\| & =\max _{t \in[0,1]} \int_{0}^{1} G(t, s) f_{p}\left(s, v(s)-u_{0}(s)\right) d s \\
& \leq \max _{t \in[0,1]} \int_{0}^{1} G(t, s) d s \phi(r)=L \phi(r) \\
& =\frac{1}{\alpha^{2}} \phi(r) \leq r .
\end{aligned}
$$

That is, $\|T v\| \leq\|v\|$ for all $v \in \mathcal{C} \cap \partial \Omega_{1}$.

Let $v \in \mathcal{C} \cap \partial \Omega_{2}$. Since $R>r$, we have $v(s)-u_{0}(s) \geq(\mu R-1) u_{0}(s) \geq 0$, $s \in[0,1]$. Then, for all $s \in[\alpha, 1-\alpha]$, we have, recalling $(2.12)$,

$$
R \geq v(s) \geq q(s)\|v\| \geq \rho R .
$$

Hence $f_{p}\left(s, v(s)-u_{0}(s)\right) \geq \psi(R)$, for $s \in[\gamma, 1-\gamma], \gamma R \leq v(s) \leq R$. Then, by (2.7) and (C),

$$
\begin{aligned}
\|T v\| & =\max _{t \in[0,1]} \int_{0}^{1} G(t, s) f_{p}\left(s, v(s)-u_{0}(s)\right) d s \\
& \geq \max _{t \in[0,1]} \int_{\gamma}^{1-\gamma} G(t, s) f_{p}\left(s, v(s)-u_{0}(s)\right) d s \\
& \geq \max _{t \in[0,1]} \int_{\gamma}^{1-\gamma} G(t, s) d s \psi(R)=C \psi(R) \\
& =\frac{1}{\alpha^{2} \sinh \alpha}(\sinh \alpha-2 \sinh \alpha \gamma \cosh \alpha / 2) \psi(R) \geq R .
\end{aligned}
$$

That is, $\|T v\| \geq\|v\|$ for all $v \in \mathcal{C} \cap \partial \Omega_{2}$.

By Theorem 1 , there exists a fixed point $v_{0} \in \mathcal{C}$ of $(2.11)$, which, equivalently, is a positive solution of the positone problem (2.10), (1.2). Moreover, $u(t)=v_{0}(t)-u_{0}(t) \geq(\mu r-1) u_{0}(t)>0$ in $[0,1]$. By Lemma $2, u$ is a positive solution of the sign-changing problem (1.1), (1.2).

The next result can be shown along similar lines.

Theorem 3. Assume that (A) and (B) hold. Suppose that there exist $r, R>0$ such that $\frac{1}{\mu}<r<R$, where $\mu>0$ satisfies (2.8), (2.9), and

(D) $\phi(R) \leq \alpha^{2} R$ and $\psi(r) \geq \frac{\alpha^{2} \sinh \alpha}{\sinh \alpha-2 \sinh \alpha \gamma \cosh \alpha / 2} r$.

Then the boundary value problem (1.1), (1.2) has at least one positive solution. 


\section{References}

[1] D. Bai and J. Yu. Semipositone problems of second order ordinary differential equations, 10 October 2009. Available from Internet: http://www.paper.edu. $\mathrm{cn} /$. Sciencepaper Online

[2] J.R. Graef and L. Kong. Positive solutions for third order semipositone boundary value problems. Appl. Math. Lett., 22:1154-1160, 2009.

http://dx.doi.org/10.1016/j.aml.2008.11.008.

[3] D. Guo and V. Lakshmikantham. Nonlinear Problems in Abstract Cones. Academic Press, Orlando, 1988.

[4] S. Li, F. Liao and H. Zhu. Multiplicity of positive solutions to second-order singular differential equations with a parameter. Bound. Value Probl., 2014(115):112, 2014. http://dx.doi.org/10.1186/1687-2770-2014-115.

[5] H. Lu. Multiple positive solutions for singular semipositone periodic boundary value problems with derivative dependence. J. Appl. Math., 2012:1-12, 2012. http://dx.doi.org/10.1155/2012/295209. Article ID 295209

[6] R. Ma. Multiple positive solutions for a semipositone fourth-order boundary value problem. Hiroshima J. Math., 33(1):217-227, 2003.

[7] A.R. Miciano and R. Shivaji. Multiple positive solutions for a class of semipositone Neumann two point boundary value problems. J. Math. Anal. Appl., 178(1):102-115, 1993. http://dx.doi.org/10.1006/jmaa.1993.1294.

[8] M.N. Nkashama and J. Santanilla. Existence of positive solutions for some nonlinear boundary value problems. J. Differential Equations, 84:148-164, 1990. http://dx.doi.org/10.1016/0022-0396(90)90131-8.

[9] H. Sua, L. Liu and Y. Wu. Positive solutions for a nonlinear second-order semipositone boundary value system. Nonlinear Anal., 71:3240-3248, 2009. http://dx.doi.org/10.1016/j.na.2009.01.201.

[10] J.P. Sun and J. Wei. Existence of positive solutions of positive solution for semipositone second-order three-point boundary-value problems. Electron. J. Differential Equations, 2008(41):1-7, 2008.

[11] Q. Yao. Positive solutions of a weak semipositone third-order three-point boundary value problem. J. Math. Res. Exposition, 30(1):173-180, 2010.

[12] L. Zhang, B. Sun and C. Xing. Existence of solutions to a third-order three-point boundary value problem. Ann. Differential Equations, 28(3):368-372, 2012.

[13] X. Zhang and L. Liu. Positive solutions of superlinear semipositone singular Dirichlet boundary value problems. J. Math. Anal. Appl., 316:525-537, 2006. http://dx.doi.org/10.1016/j.jmaa.2005.04.081. 